\title{
Diaphragmatic Rupture and Gastric Perforation in a Patient with COVID-19 Pneumonia
}

\author{
Erika Poggiali*, Andrea Vercelli*, Elena Demichele, Eva loannilli, Andrea Magnacavallo \\ Emergency Department, Guglielmo da Saliceto Hospital, Piacenza, Italy \\ *these authors contributed equally to this work
}

Received: 20/05/2020

Accepted: 25/05/2020

Published: 27/05/2020

How to cite this article: Poggiali E, Vercelli A, Demichele E, loannilli E, Magnacavallo A. Diaphragmatic rupture and gastric perforation in a patient with COVID-19 pneumonia. EJCRIM 2020;7: doi:10.12890/2020_001738.

Conflicts of Interests: The Authors declare that there are no competing interests.

Acknowledgements: The authors thank all staff of the emergency department of their hospital for their strength, high levels of involvement, responsibility, accountability, flexibility, care and dedication to their work at such a difficult time for the Italian healthcare system.

This article is licensed under a Commons Attribution Non-Commercial 4.0 License

\section{ABSTRACT}

We describe the case of a young female patient admitted to our emergency department during the Italian COVID-19 epidemic, for fever and dry cough associated with symptoms of gastric reflux over the previous 5 days. Lung ultrasound showed diffuse bilateral B lines with irregular pleural thickening, and consolidation with air bronchogram and slight pleural effusion in the lower left lobe. Chest HRCT and abdominal CT scanning with contrast revealed diaphragmatic rupture with gastric perforation, and atelectasis of the left pulmonary lobe with unilateral pleural effusion, diffuse ground-glass opacities and multiple small consolidations in both lobes. A nasopharyngeal swab for 2019-nCoV was positive. A diagnosis of diaphragmatic rupture and gastric perforation in COVID-19 pneumonia was made. The patient was immediately hospitalized and surgically treated. Treatment for COVID-19 and empiric antibiotic therapy were promptly started.

\section{LEARNING POINTS}

- Coronavirus disease (COVID-19) can cause fever, dry cough and acute respiratory failure.

- Cough can result in several complications, including rupture of the diaphragm and abdominal herniation.

- CT scanning is the gold standard technique to investigate COVID-19 pneumonia and diaphragmatic rupture.

\section{KEYWORDS}

COVID-19, pneumonia, gastric perforation, diaphragmatic rupture, acute chest pain

\section{CASE DESCRIPTION}

A 54-year-old female patient was admitted to our emergency department during the COVID-19 epidemic complaining of fever and dry cough with symptoms of gastric reflux disease (sore throat and a burning feeling in her throat) over the previous 5 days. She had a medical history of hypertension treated with an ACE inhibitor (ramipril $2.5 \mathrm{mg}$ every 8 hours) and a beta-blocker (metoprolol 100 mg every 12 hours), and previous episodes of nephrolithiasis which always resolved with medical treatment. The patient did not have any harmful habits or chronic pulmonary disease.

At admission the patient had severe acute chest pain. Physical examination revealed a normal BMI, blood pressure and body temperature, but increased heart and respiratory rates of 110 beats/minute and 22 breaths/minute, respectively. The abdomen was painful, but soft with no palpable mass. An electrocardiogram showed a regular sinus rhythm with normal ventricular repolarization. 
Blood arterial gas analysis revealed respiratory alkalosis and hypoxaemia $\left(\mathrm{PaO}_{2} / \mathrm{FiO}_{2} 250 \mathrm{mmHg}\right)$, and a high $\mathrm{A}$-a gradient (61.3 mmHg instead of the $17.5 \mathrm{mmHg}$ expected for age) suggesting an oxygen transfer/gas exchange problem. The patient was treated with high-flow oxygen, paracetamol (1 g iv) and morphine sulfate (repeated iv bolus of 2-3 mg) for the management of severe pain, and intravenous fluid therapy with crystalloid solution.

Point-of-care lung ultrasound showed diffuse bilateral B lines with irregular pleural thickening, and consolidation with air bronchogram and slight pleural effusion in the lower left lobe ${ }^{[1]}$.

HRCT of the chest and abdominal CT scanning with contrast were performed and showed diaphragmatic rupture (Figure $1 \mathrm{~A}$ ) with gastric herniation and perforation (Figure 1B), causing left pulmonary collapse with unilateral pleural effusion (antero-posterior diameter 20 mm); the remaining pulmonary parenchyma of both lobes presented diffuse ground-glass opacities and multiple small consolidations (Figure 1C), as reported in COVID-19 pneumonia ${ }^{[2,3]}$. No rib fractures were detected.

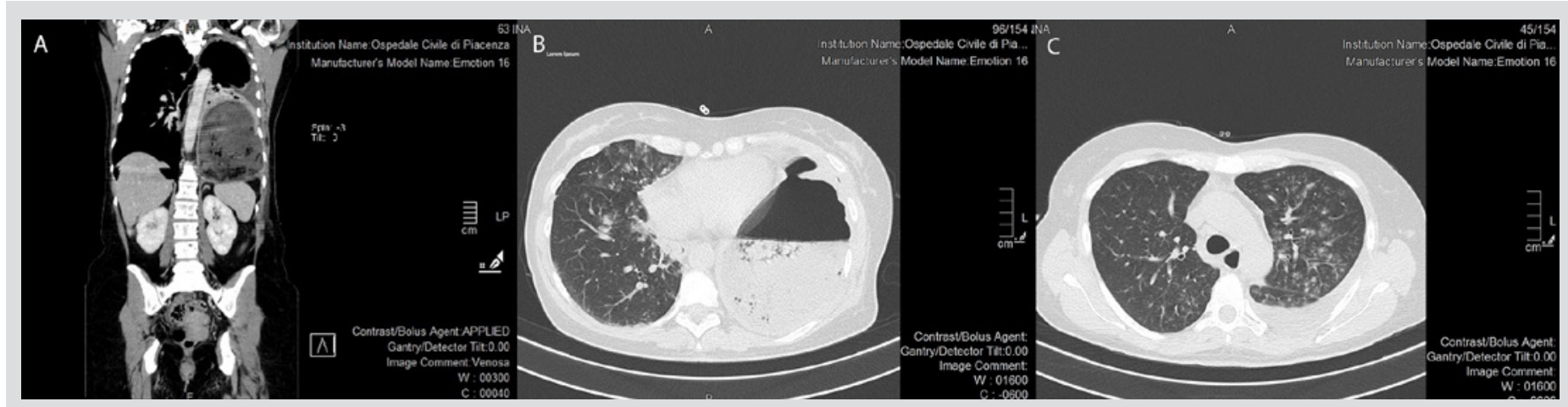

Figure 1. Diaphragmatic rupture (Panel A, coronal view) with gastric perforation (Panel B, axial view). Atelectasis of the left pulmonary lobe with pleural effusion (anteroposterior diameter $20 \mathrm{~mm}$ ); diffuse ground-glass opacities and multiple small consolidations are evident in the remaining pulmonary parenchyma of both lobes (Panel C).

\section{DISCUSSION}

COVID-19 can present with different clinical scenarios ranging from flu-like symptoms with fever, dry cough and sore throat to gastrointestinal issues to severe acute respiratory distress syndrome (ARDS) and multi-organ failure with death. Cough can be associated with rib fracture, abdominal herniation and diaphragmatic rupture ${ }^{[5,6]}$. The most common complication of cough is rib fracture ${ }^{[7]}$. Rarely, patients can present with serious complications such as pneumothorax, bleeding or intercostal pulmonary hernia. Diaphragmatic rupture can occur following intensive coughing, and causing the herniation of bowel loops into the chest. As a consequence, the patient may complain of chest and abdominal pain, which can mimic an acute abdomen, appendicitis or gynaecological disease. Abdominal organ herniation into the chest can impair ventilation and oxygen delivery. This was an extremely serious situation in our patient due to her concomitant COVID-19 pneumonia.

\section{CONCLUSIONS}

To the best of our knowledge, this is the first case of diaphragmatic rupture and gastric herniation in a patient with COVID-19 pneumonia to be reported the literature. The incidence of abdominal organ herniation due to diaphragmatic rupture is unknown since many cases likely go undiagnosed or are diagnosed late. Diaphragmatic rupture is a life-threatening condition which need prompt diagnosis and repair. CT scanning is the gold standard technique to evaluate the diaphragm ${ }^{[8]}$.

Our experience emphasizes the importance of maintaining focus on the patient's symptoms and the prominent role of lung ultrasound during the COVID-19 epidemic.

As reported in the literature, we continue to learn about the clinical manifestations of COVID-19 and its complications in patients with the disease. 


\section{REFERENCES}

1. Poggiali E, Dacrema A, Bastoni D, Tinelli V, Demichele E, Mateo Ramos P, et al. Can lung ultrasound help critical care clinicians in the early diagnosis of novel coronavirus (COVID-19) pneumonia? Radiology 2020;295(3):E6.

2. Feng P, Tianhe Y, Peng S, Shan G, Bo L, Lingli L, et al. The course of lung changes on chest CT during recovery from 2019 novel coronavirus (COVID-19) pneumonia. Radiology 2020;295(3):715-721.

3. Chung M, Bernheim A, Mei X, Zhang N, Huang M, Zeng X, et al. CT imaging features of 2019 novel coronavirus (2019-nCoV). Radiology 2020;295(1):202-207.

4. Lippi G, Mattiuzzi C, Bovo C, Plebani M. Current laboratory diagnostics of coronavirus disease 2019 (COVID-19). Acta Biomed 2020;91(2):137-145.

5. Hillenbrand A, Henne-Bruns D, Wurl P. Cough induced rib fracture, rupture of the diaphragm and abdominal herniation. World J Emerg Surg 2006;1:34.

6. Farinacci-Vilaró M, Gerena-Montano L, Nieves-Figueroa H, Garcia-Puebla J, Fernández R, Hernández R, et al. Chronic cough causing unexpected diaphragmatic hernia and chest wall rupture. Radiol Case Rep 2020;15(1):15-18.

7. Roberge RJ, Morgenstern MJ, Osborn H. Cough fracture of the ribs. Am J Emerg Med 1984:2:513-517.

8. Shanmuganathan K, Killeen K, Mirvis SE, White CS. Imaging of diaphragmatic injuries. J Thorac Imaging 2000;15:104-111. 